\title{
Probability density functions of turbulent velocity and temperature in the atmospheric surface layer
}

\author{
Chia R. Chu, ${ }^{1,2}$ Marc B. Parlange, ${ }^{1}$ Gabriel G. Katul, ${ }^{3}$ and John D. Albertson ${ }^{1}$
}

\begin{abstract}
The probability density functions (pdf's) for the longitudinal and vertical velocities, temperature, their derivatives, and momentum and sensible heat fluxes were measured in the atmospheric surface layer for a wide range of atmospheric stability conditions. The measured pdf's for both the velocity and the temperature fluctuations are near-Gaussian and consistent with corresponding laboratory measurements for nearneutral and stable stability conditions. Hence the first- and second-order moments are sufficient to predict the heat and momentum flux pdf's. The lower-order moments can be estimated from mean meteorological conditions using surface layer similarity theory. For unstable conditions the pdf for temperature is non-Gaussian and is strongly skewed due to local convective thermal plumes. For near-neutral and stable conditions the pdf's for the velocity and temperature longitudinal gradients have long exponential tails, in agreement with findings in laboratory experiments and numerical simulations.
\end{abstract}

\section{Introduction}

It is known that atmospheric turbulent transport mechanisms are responsible for the removal of heat and water vapor from the ground into the atmosphere. The turbulent flow variables responsible for these transport mechanisms are governed by fluctuations that are quasi-random [Monin and Yaglom, 1971]. In hydrology, Monin and Obukhov [1954] surface layer similarity theory has been successfully used to describe the mean quantities as well as second-order moments of these fluctuations [Brutsaert, 1982; Kader and Yaglom, 1990]. However, to characterize more complex quantities, such as surface fluxes of momentum, heat, and water vapor transport, higherorder moments and cumulants are required [Raupach et al., 1991].

In the statistical theory of turbulence the probability density functions (pdf's) provide a complete probabilistic description that permits the estimation of moments and cumulants of land surface fluxes, velocity, and scalars. Thus far the velocity and scalar pdf's have been used to (1) better quantify physical transport mechanisms [Caughey et al., 1983], (2) construct closure models for the time-averaged Navier-Stokes equations, and (3) develop phenomenological models [Pope, 1985; Lesieur, 1990].

As discussed by Townsend [1947], for homogeneous isotropic turbulent flows the velocity pdf's are near-Gaussian due to the central limit theorem (CLT). Thus, in many turbulence models, it was assumed, a priori, that the scalar pdf is also Gaussian [see Batchelor, 1953]. More recently, direct numerical simulations and improved experimental techniques have permitted detailed examination of the velocity and scalar pdf's.

The numerical simulations were used to investigate the re-

\footnotetext{
${ }^{1}$ Hydrologic Sciences, University of California, Davis.

${ }^{2}$ Department of Civil Engineering, National Central University, Chung-Li, Taiwan.

${ }^{3}$ School of the Environment, Duke University, Durham, North Carolina.

Copyright 1996 by the American Geophysical Union.

Paper number 96WR00287.

0043-1397/96/96WR-00287\$09.00
}

lationship between scalar and velocity pdf's for homogenous and isotropic turbulence. For example, Kerr [1985] found that the kurtosis (or flatness factor) for the scalar derivative is larger than the velocity derivative for isotropic turbulence, suggesting that the scalar field is more intermittent than the velocity field. Using direct and large-eddy simulations, Metais and Lesieur [1992] found that for stably stratified homogeneous decaying turbulence, the pdf's of velocity and scalars exhibit near-Gaussian behavior, while the pdf's of scalar gradients have long exponential tails.

A number of laboratory experiments were also carried out to quantify the scalar pdf and its relationship to the velocity pdf's for nonisotropic turbulent flows. Thoroddsen and Van Atta [1992] found that the pdf's for the temperature gradients exhibit extended exponential tails, while those for temperature fluctuations are nearly Gaussian and similar to the velocity field for stable stability conditions. Jayesh and Warhaft [1992] considered the spatial evolution of the temperature pdf's in grid-generated turbulence for a wide range of Reynolds numbers. Their results showed that the temperature pdf exponential tails are dependent on the magnitude of the Reynolds number. Pumir et al. [1991] developed a one-dimensional phenomenological model for a passive scalar randomly advected by turbulence and showed that exponential tails are generic to scalar fluctuations if the mixing of scalar is subject to a mean scalar gradient. It appears from the above laboratory studies that the velocities may have Gaussian pdf's for near-neutral or slightly stable stability; however, the scalar and the velocity gradient pdf's depart from Gaussian and exhibit strong exponential tails.

Thus far the relation between the scalar and velocity pdf's, as well as pdf's of certain dynamical quantities such as the fluxes and gradients, has focused on laboratory and numerical simulations that may not represent the conditions in the atmospheric surface layer (ASL). In this study we examine the pdf's of velocities, temperature, and their derivatives and fluxes measured in the ASL under a wide range of stability conditions. Also, we investigate the possibility of using surface layer similarity theory to predict the pdf's of momentum and sensible heat fluxes for near-neutral and stable stability conditions. 

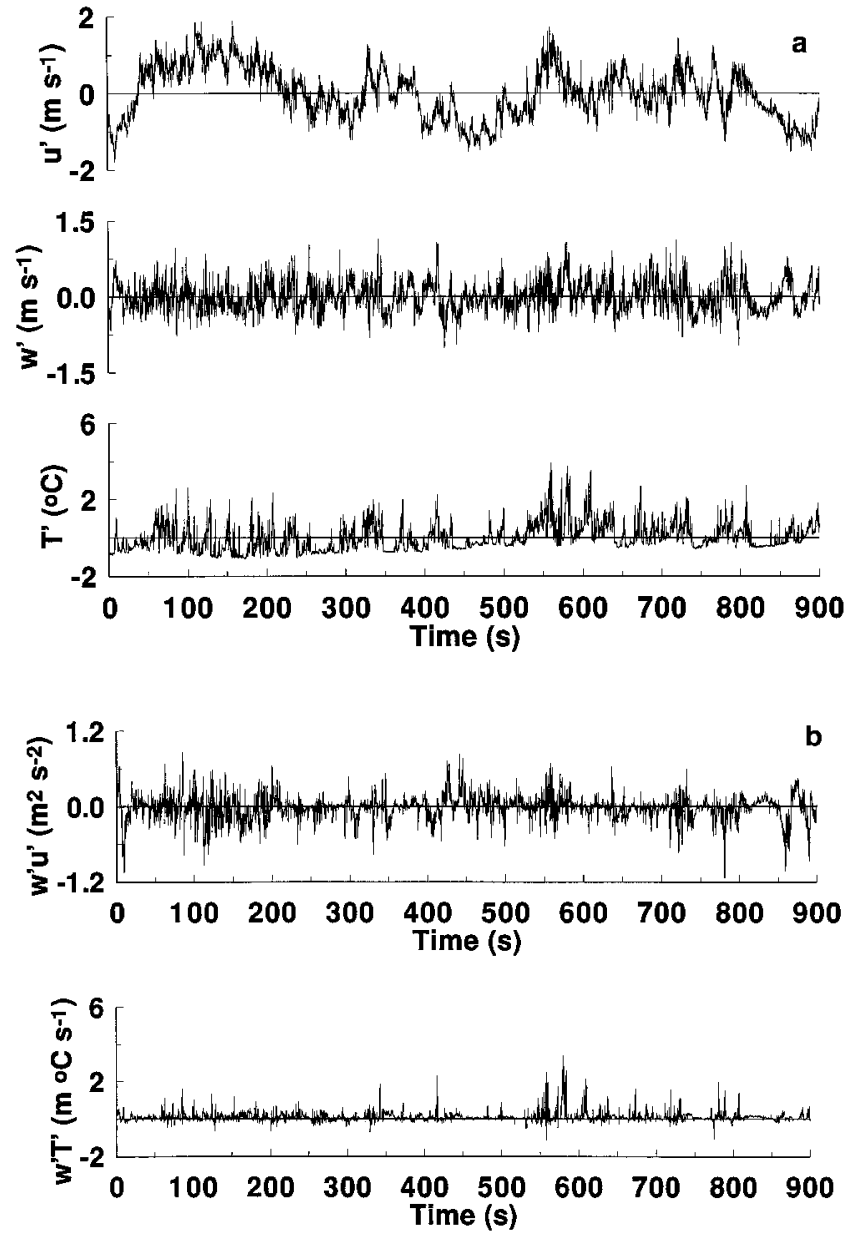

Figure 1. (a) The measured time variation of temperature $\left(T^{\prime}\right)$ and longitudinal $\left(u^{\prime}\right)$ and vertical $\left(w^{\prime}\right)$ velocity fluctuations for unstable atmospheric conditions. (b) Same as Figure 1a but for heat $\left(w^{\prime} T^{\prime}\right)$ and momentum $\left(u^{\prime} w^{\prime}\right)$ fluxes.

The existence of such a relationship provides an operational tool to characterize the variability in momentum and sensible heat fluxes from routinely measured mean meteorological quantities.

\section{Experimental Setup}

The experiment was carried out in June 1993 over the dry Owens Lake bed in Owens Valley, California. The lake bed is part of a large basin bounded by the Sierra Nevada range and the White and Inyo Mountains. The experimental site is 1100 $\mathrm{m}$ above sea level and is located on the northeast section of the lake bed. The fetch surrounding the instrumented site is uniform for several kilometers. The surface is crusty sand with a mean surface roughness length $z_{o}$ of $0.13 \mathrm{~mm}$; however, the variation around this estimate is large [see Katul, 1994; Albertson et al., 1995].

The longitudinal, lateral, and vertical velocity components ( $U=U_{1}, V=U_{2}$, and $W=U_{3}$ ) were measured using a triaxial ultrasonic anemometer (Gill Instruments/1012R2). In this study, both meteorological and index notations are used. The sampling frequency $f_{s}$ and recording period were $56 \mathrm{~Hz}$ and $15 \mathrm{~min}$, respectively, resulting in 50,400 measurements per velocity component. The short sampling period was necessary to ensure steady state in the mean meteorological conditions. The mean meteorological parameters such as net radiation, soil heat flux, surface temperature, mean wind speed, and wind direction were also measured during the experiment [see Katul, 1994; Katul et al., 1995a, b; Albertson et al., 1995].

The air temperature $T_{a}$ was determined from the speed of sound $C$ using

$$
T_{a}=\frac{C^{2}}{\alpha R_{d}}
$$

where $R_{d}\left(=287.04 \mathrm{~J} / \mathrm{Kg} /{ }^{0} \mathrm{~K}\right)$ is the gas constant of dry air and $\alpha(=1.4)$ is the ratio of the molar specific heat capacities of air at constant pressure to that at constant volume. The adequacy of the Gill triaxial sonic anemometer to measure temperature fluctuations is discussed by Katul [1994] and Katul et al. [1995a, b]. The digital signals from the Gill ultrasonic anemometer were logged directly to a 486 portable computer and stored in 15-min files on the hard drive for future processing. A coordinate rotation was applied to each 15-min file so that $\left\langle U_{2}\right\rangle=\left\langle U_{3}\right\rangle=0$, where $\left\langle U_{1}\right\rangle$ is aligned along the longitudinal direction, and angle brackets denote the time averaging operation. A sample output of the $u^{\prime}=U-\langle U\rangle, w^{\prime}=W-$ $\langle W\rangle$, and $T^{\prime}=T_{a}-\left\langle T_{a}\right\rangle$ time series and the momentum $\left(u^{\prime} w^{\prime}\right)$ and sensible heat $\left(w^{\prime} T^{\prime}\right)$ fluxes are given in Figures 1a and $1 \mathrm{~b}$, respectively. Notice in Figure 1a the ramp-like pattern in the $T^{\prime}$ time series and in Figure 1b the strong intermittent nature of the heat and momentum fluxes.

Three runs with different stability conditions were chosen to investigate the shape of pdf's for the velocity and scalar fluctuations. The mean meteorological conditions for these runs are summarized in Table 1 . The friction velocity $u_{*}\left(=\left\langle-u^{\prime} w^{\prime}\right\rangle^{1 / 2}\right)$ and sensible heat flux $H=\rho c_{p}\left\langle w^{\prime} T^{\prime}\right\rangle$ were calculated from the three-dimensional (3-D) sonic anemometer measurements, where $\rho$ is the density of air and $c_{p}\left(=1005 \mathrm{~J} /{ }^{0} \mathrm{~K}\right)$ is the specific heat of air under constant pressure. The Obukhov length $L$ was calculated

$$
L=-\rho u_{*}{ }^{3} / \kappa g \frac{H}{\left\langle T_{a}\right\rangle C_{p}}
$$

where $\kappa(=0.4)$ is the von Karman constant and $g$ is the gravitational acceleration. In (2) the bouyancy correction due to water vapor was neglected [see Albertson et al., 1995].

This ASL study differs from many laboratory experiments and numerical simulations in that (1) the Reynolds numbers are at least 5 times larger, (2) the turbulence is influenced by both shear and buoyancy forces, and (3) the separation between production and dissipation scales is five decades.

Table 1. Summary of Mean Meteorological Conditions

\begin{tabular}{lccc}
\hline \multicolumn{1}{c}{ Stability Conditions } & Neutral & Stable & Unstable \\
\hline Day of Year & 179 & 180 & 178 \\
Time, UT & 2134 & 0602 & 0853 \\
Height $z, \mathrm{~m}$ & 2.0 & 2.5 & 3.0 \\
Mean velocity $\langle U\rangle, \mathrm{m} / \mathrm{s}$ & 6.49 & 2.89 & 1.87 \\
Air temperature $T_{a},{ }^{\circ} \mathrm{C}$ & 25.4 & 16.6 & 27.5 \\
Surface temperature $T_{s},{ }^{\circ} \mathrm{C}$ & 19.8 & 11.5 & 37.7 \\
Shear velocity $u^{*}, \mathrm{~m} / \mathrm{s}$ & 0.255 & 0.096 & 0.144 \\
Obukhov length $L, \mathrm{~m}$ & 35.3 & 6.56 & -2.51 \\
Sensible heat flux $H, \mathrm{~W} / \mathrm{m}^{2}$ & -37.6 & -10.8 & 94.9 \\
\hline
\end{tabular}




\section{Results and Discussion}

This section is divided into four parts, covering (1) pdf's of velocity and temperature, (2) pdf's of velocity and temperature longitudinal gradients, (3) joint pdf's of vertical velocity and temperature, and (4) pdf's of momentum and sensible heat fluxes. The use of similarity theory to predict the parameters for the pdf's of fluxes is presented.

The measured pdf's were computed from the discrete frequency distribution of the velocity, temperature, their derivatives, and momentum and sensible heat fluxes. For each flow variable this frequency distribution was generated by clustering the 50,400 measurements within 500 bins. Notice that the measured pdf extends for three to four decades and hence is suitable for investigating the pdf tails and the contributions from extreme events.

\subsection{PDFs of Velocity and Temperature}

The probability density functions for the horizontal velocity $u^{\prime}$, vertical velocity $w^{\prime}$, and temperature $T^{\prime}$ fluctuations for different stability conditions are shown in Figures $2 a, 2 b$, and $2 c$, respectively. The lower two curves have been shifted down one and two decades for clarity in the comparisons. In order to better contrast departures from Gaussian distributions for all stability conditions, these pdf's are normalized using

$$
P(X)=P\left(\frac{X-\langle X\rangle}{\sigma_{X}}\right)
$$

where $\sigma_{X}$ and $\langle X\rangle$ are the standard deviation and mean of the measured flow variable $X$, respectively. The solid lines in Figure 2 are zero-mean and unit variance Gaussian pdf's. To accentuate the pdf tails in Figures 2, a log ordinate axis is used. Notice that the exponential tails are linear using this graphical representation. For a quantitative comparison with the Gaussian distribution, the skewness $\left(S=(X-\langle X\rangle)^{3} / \sigma_{X}^{3}\right)$ and kurtosis $\left(K=(X-\langle X\rangle)^{4} / \sigma_{X}^{4}\right)$ factors for all the variables are reported in Table 2. Under neutral stratification (Figure 2a), fluctuations of velocities $\left(u^{\prime}\right.$ and $\left.w^{\prime}\right)$ and temperature $\left(T^{\prime}\right)$ were all close to Gaussian (i.e., $S=0, K=3$ ). However, under stable condition (Figure $2 \mathrm{~b}$ ), the vertical velocity fluctuations deviate from Gaussian, given the high kurtosis $(K=$ 5.78). This is due to the intermittent structure of stably stratified atmospheric flow and the possible effects of gravity waves [Kaimal and Finnigan, 1994]. For unstable stratification the temperature pdf (Figure 2c) was skewed toward positive fluctuations $(S=1.12, K=5.36)$; yet the vertical velocity remains nearly Gaussian-distributed $(S=0.31, K=3.12)$. This skewed distribution of the temperature pdf is similar to that obtained from simulations by Balachandar and Sirovich [1991] for Rayleigh-Benard turbulent convection near the boundary. The skewness is due to the abrupt increase in temperature caused by the upward thermal plumes in strong convective flows.

\subsection{PDFs of Longitudinal Gradients}

While the tails of pdf's for flow variables are influenced by large-scale eddy motion (i.e., associated with large perturba-

Figure 2. (Opposite) Probability density functions under different stability conditions: (a) horizontal velocity, (b) vertical velocity, and (c) temperature. The solid lines are Gaussian, a circle denotes a neutral case, a square denotes a stable case, and a triangle denotes an unstable case.
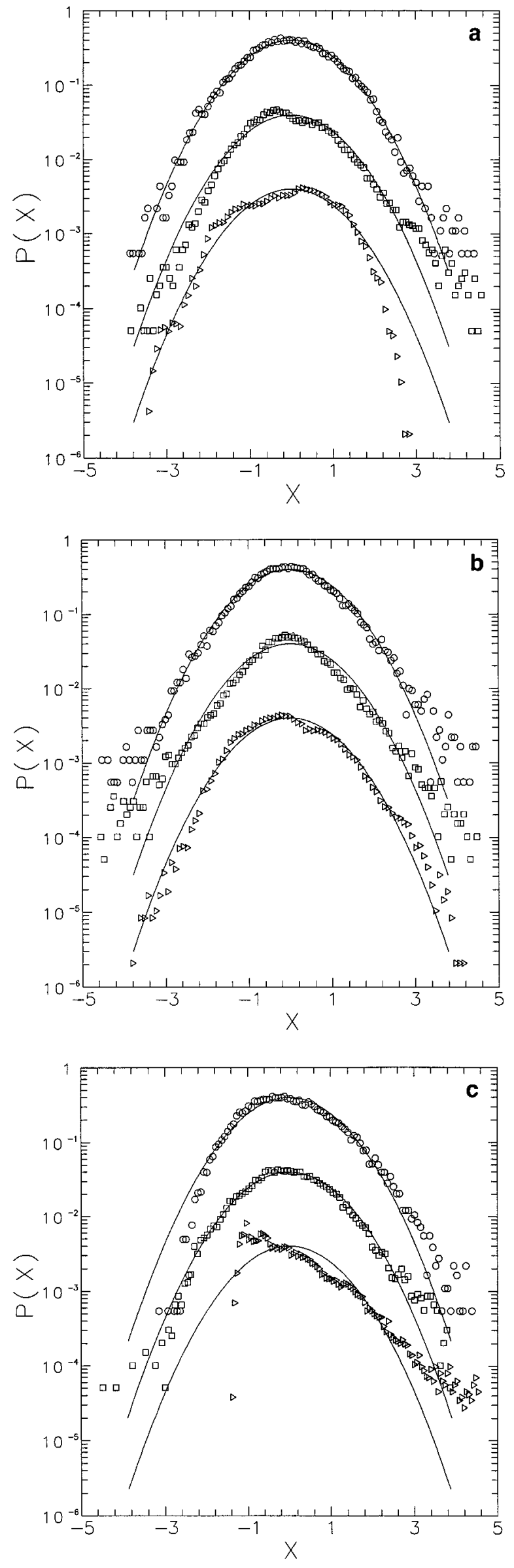

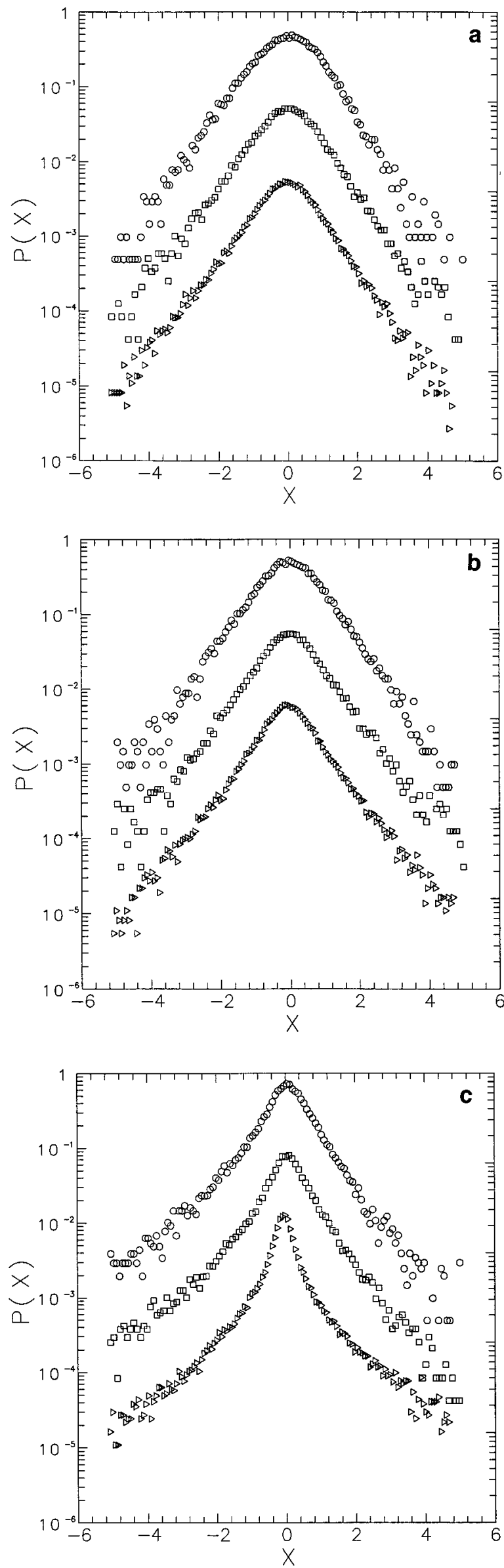

Table 2. Summary of Skewness $(S)$ and Kurtosis $(K)$ Factors for Various Turbulent Quantities

\begin{tabular}{|c|c|c|c|c|c|c|}
\hline \multirow[b]{2}{*}{ Variable } & \multicolumn{2}{|c|}{ Neutral } & \multicolumn{2}{|c|}{ Stable } & \multicolumn{2}{|c|}{ Unstable } \\
\hline & $S$ & K & $S$ & K & $S$ & $K$ \\
\hline$u^{\prime}$ & 0.08 & 3.11 & 0.16 & 3.95 & -0.19 & 2.47 \\
\hline$w^{\prime}$ & 0.14 & 3.77 & 0.06 & 5.78 & 0.31 & 3.12 \\
\hline$T^{\prime}$ & 0.24 & 3.10 & 0.04 & 3.37 & 1.12 & 5.36 \\
\hline$d u / d x$ & -0.23 & 4.47 & -0.21 & 5.66 & -0.30 & 6.84 \\
\hline$d w / d x$ & -0.11 & 5.45 & -0.16 & 7.70 & 0.03 & 8.29 \\
\hline$d T / d x$ & -0.95 & 10.2 & -1.13 & 12.8 & 0.82 & 27.3 \\
\hline$u^{\prime} w^{\prime}$ & -0.03 & 3.93 & -0.35 & 7.43 & 0.15 & 3.86 \\
\hline$w^{\prime} T^{\prime}$ & 0.11 & 3.78 & -0.09 & 6.01 & 0.36 & 3.23 \\
\hline
\end{tabular}

tions from the mean), the tails of gradient pdf's are governed by short-lived eddy motion that departs significantly from the mean gradient. The normalized pdf's of the longitudinal gradients for horizontal velocity $\left(d u^{\prime} / d x\right)$, vertical velocity $\left(d w^{\prime} / d x\right)$ and temperature $\left(d T^{\prime} / d x\right)$ are presented in Figures $3 \mathrm{a}, 3 \mathrm{~b}$, and $3 \mathrm{c}$, respectively. The skewness and kurtosis factors for these pdf's are given in Table 2. To determine these spatial gradients from time series measurements, Taylor's [1938] hypothesis was used $(d x=-\langle U\rangle d t)$. Although the application of Taylor's [1938] frozen turbulence hypothesis introduces some distortions at high wavenumbers due to the variability in the longitudinal velocity, this distortion is small if the squared turbulence intensity $I_{u}^{2}\left(=\left[\sigma_{u} /\langle U\rangle\right]^{2}\right)$ does not exceed 0.1 [see Wyngaard and Clifford, 1977]. As evidenced from Tables 1 and 3, the measured $I_{u}^{2}$ does not exceed 0.1 for all three runs.

For an exponential pdf distribution the pdf is of the form

$$
P(X) \propto \exp (-D|X|)
$$

Note that in Figures 3a, 3b, and 3c the normalized pdf's for the velocity gradients have very similar shapes for all three stability conditions. Hence, for the velocity fluctuations, atmospheric stability has minimal effect on the slopes of the tails. In Table 2 the kurtosis for the velocity gradients are close to 6 and are also not sensitive to $z / L$. The kurtosis factor of an exponential pdf is 6. However, the pdf's for $d T^{\prime} / d x$ are dependent on atmospheric stability conditions. For unstable conditions, $d T^{\prime} / d x$ (Figure 3c) shows an extruded peak for small temperature differences; such a peak is not formed for neutral and stable stability conditions. Similar shapes have been reported for the scalar gradients in laboratory experiments by Antonia et al. [1984] and Thoroddsen and Van Atta [1992]. Also notice that for unstable conditions the kurtosis for $d u^{\prime} / d x$ is comparable to that of $T^{\prime}$ (Table 2). As in the Metais and Lesieur [1992] large-eddy simulation results, the similarity in the $d u^{\prime} / d x$ and $T^{\prime}$ pdf's is consistent with the working hypothesis that the temperature field is being strained by the velocity field.

For unstable conditions the edges of the ramp-like pattern in the temperature time series (see, e.g., Figure 1a) produce large temperature gradients that contribute to the tails in Figure 3c. The ramp-like pattern is absent for stable and neutral stability conditions. Thus the tails of temperature gradient pdf's for the unstable ASL are governed by the ramp-like motion [see also

Figure 3. (Opposite) Probability density functions of the longitudinal derivatives under different stability conditions: (a) horizontal velocity, (b) vertical velocity, and (c) temperature. Conditions and symbols are the same as Figure 2. 
Katul et al., 1994]. This is also confirmed by the large kurtosis factor (27.3) for unstable conditions vis-a-vis the near-neutral (10.2) and stable conditions (12.8) (see Table 2).

\subsection{Joint PDF of Vertical Velocity and Temperature}

The joint probability distribution for the normalized vertical velocity and temperature fluctuations under neutral, stable, and unstable stability conditions are shown in Figures $4 a, 4 b$, and $4 \mathrm{c}$, respectively. For neutral and stable conditions the pdf is symmetrical in the diagonal direction. For unstable conditions (Figure 4c), a large asymmetry is noted. Qualitatively, this asymmetry is similar to the laboratory study of turbulent convection by Adrian et al. [1986] and is attributed to the enhanced upward transport of heat due to the bursting thermal plumes near the surface. As the thermal plumes move upward, the temperature of the plume decreases as it mixes with the surrounding cold air and produces the ramp-like pattern noted in Figure 1a. The rapid temperature drop causes the plume to decelerate and produces an asymmetric vertical velocity perturbation. Notice that the probability mass is concentrated in the first and third quadrants (Figure 4c), indicating that the sensible heat flux is dominated by this ramp-like structure.

\subsection{PDF of the Fluxes}

The pdf's for the fluctuations of momentum $u^{\prime} w^{\prime}$ and sensible heat $w^{\prime} T^{\prime}$ fluxes are considered here. The fluctuations of momentum and sensible heat fluxes are computed from the instantaneous product of $u^{\prime}$ and $w^{\prime}$, and $w^{\prime}$ and $T^{\prime}$, respectively. Figures $5 \mathrm{a}$ and $5 \mathrm{~b}$ show the pdf's of momentum and sensible heat fluxes, respectively. These pdf's all exhibit asymmetric shape due to uneven distributions of positive and negative fluxes. Notice that for the stable condition the intermittent structure of vertical velocity affects both fluxes, resulting in high kurtosis factors (see Table 2).

Since the $u^{\prime}, w^{\prime}$, and $T^{\prime}$ pdf's are close to Gaussian for neutral and stable conditions, we investigate the behavior of their product by assuming a jointly Gaussian pdf model. The probability density function of two jointly Gaussian random variables $x$ and $y$ can be written as

$$
\begin{aligned}
p(x, y)= & \frac{1}{\pi \sigma_{x} \sigma_{y}\left(1-r^{2}\right)^{1 / 2}} \\
& \cdot \exp \left[\frac{1}{2\left(1-r^{2}\right)}\left(\frac{x^{2}}{\sigma_{x}^{2}}-\frac{2 r x y}{\sigma_{x} \sigma_{y}}+\frac{y^{2}}{\sigma_{y}^{2}}\right)\right]
\end{aligned}
$$

where $r\left(=\langle x y\rangle / \sigma_{x} \sigma_{y}\right)$ is the correlation coefficient of $x$ and $y$ [Papoulis, 1972; Thoroddsen and Van Atta, 1992]. The cumulative distribution $P(z)$ for the product of $x$ and $y(z=x y)$ is given by

$$
P_{z}(z)=\int_{-\infty}^{\infty} \int_{-\infty}^{z / y} p(x, y) d x d y
$$

As discussed by Thoroddsen and Van Atta [1992], the probability density function $p(z)$ can be derived from (6) using

Figure 4. (Opposite) Joint probability density for vertical velocity and temperature fluctuations under different stability conditions: (a) neutral condition, (b) stable condition, and (c) unstable condition.
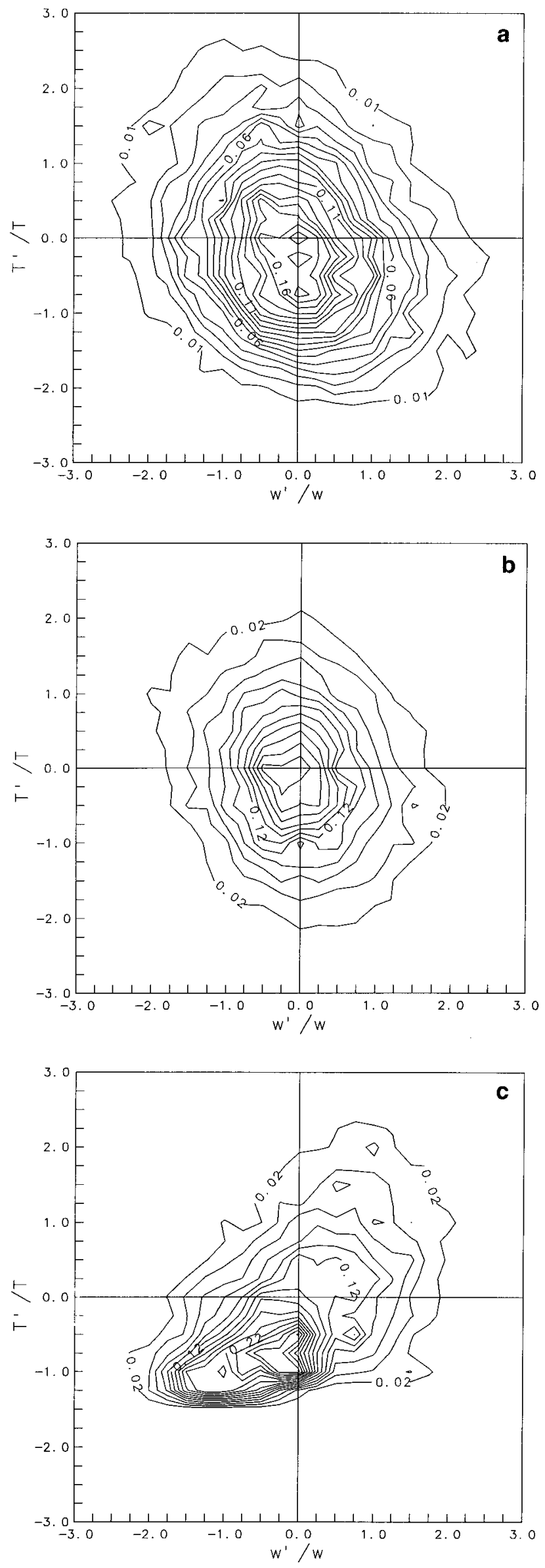
Table 3. Comparison of Measured and Predicted Parameters for the PDFs Statistics

\begin{tabular}{|c|c|c|c|c|c|c|}
\hline \multirow{2}{*}{$\begin{array}{c}\text { Stability } \\
\text { Condition }\end{array}$} & \multicolumn{3}{|c|}{ Measured } & \multicolumn{3}{|c|}{ Predicted } \\
\hline & Neutral & Stable & Unstable & Neutral & Stable & Unstable \\
\hline$u^{*}, \mathrm{~m} / \mathrm{s}$ & 0.255 & 0.096 & 0.144 & 0.270 & 0.095 & 0.134 \\
\hline$H, \mathrm{~W} / \mathrm{m}^{2}$ & -37.6 & -10.8 & 94.9 & -60.9 & -17.5 & 88.0 \\
\hline$\sigma_{u}, \mathrm{~m} / \mathrm{s}$ & 0.74 & 0.41 & 0.67 & 0.69 & 0.27 & $\ldots$ a \\
\hline$\sigma_{w}, \mathrm{~m} / \mathrm{s}$ & 0.36 & 0.13 & 0.27 & 0.32 & 0.13 & 0.29 \\
\hline$\sigma_{T}, \mathrm{C}$ & 0.47 & 0.60 & 0.71 & 0.42 & $\ldots$ & 0.59 \\
\hline$r_{u w}$ & -0.25 & -0.17 & -0.12 & -0.29 & -0.14 & $\cdots$ \\
\hline$r_{w T}$ & -0.22 & -0.13 & 0.48 & -0.27 & $\ldots$ & 0.45 \\
\hline
\end{tabular}

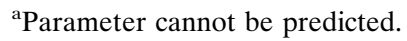

$$
p_{z}(z)=2 \int_{0}^{\infty} \frac{1}{y} f_{x y}\left(\frac{z}{y}, y\right) d y
$$

Substituting (5) into (7), the resulting distribution becomes

$$
\begin{aligned}
p(x, y)= & \frac{1}{2 \pi \sigma_{x} \sigma_{y}\left(1-r^{2}\right)^{1 / 2}} \exp \left(\frac{r z}{\left(1-r^{2}\right) \sigma_{x} \sigma_{y}}\right) \\
& \cdot \int_{0}^{\infty} \frac{1}{y} \exp \left[-\frac{1}{2}\left(y+\frac{w^{2}}{y}\right)\right] d y
\end{aligned}
$$

where $w=z /\left(\left(1-r^{2}\right) \sigma_{x} \sigma_{y}\right)$. The integration yields

$$
\begin{aligned}
p(x, y)= & \frac{1}{\pi \sigma_{x} \sigma_{y}\left(1-r^{2}\right)^{1 / 2}} \exp \left(\frac{r z}{\left(1-r^{2}\right) \sigma_{x} \sigma_{y}}\right) \\
& \cdot K_{o}\left(\frac{|z|}{\sigma_{x} \sigma_{y}\left(1-r^{2}\right)}\right)
\end{aligned}
$$

where $K_{o}$ is the modified Bessel function of the second kind. This result was originally derived and tested by Thoroddsen and Van Atta [1992] and was further evaluated for the near-neutral and slightly stable ASL by Katul [1994]. The solid lines in Figures $5 \mathrm{a}$ and $5 \mathrm{~b}$ are the predictions from (9). The predicted values were not plotted for fluxes approaching zero, since the modified Bessel function has a logarithmic singularity at the origin. For the momentum fluxes (Figure 5a), good agreement between predictions and measurements are noted due to the near-Gaussian distribution of the velocities.

For the sensible heat flux under unstable conditions (Figure $5 b)$, the agreement between measured and predicted pdf's from (9) does not agree well for positive sensible heat fluxes, especially at the tails. The failure of the prediction is attributed to the skewed distribution of temperature fluctuations noted in Figure 2c.

This analysis suggests that the Gaussian model can describe the pdf's of velocities, temperature, and fluxes under neutral and stable conditions. Since only three second-order moments are needed to describe the pdf, Monin and Obukhov [1954] similarity theory can be used to estimate these pdf parameters [see also Katul, 1994]. The use of Monin and Obukhov [1954] similarity theory permits the estimation of the pdf flux parameters from routinely measured mean meteorological variables. From Monin and Obukhov [1954] surface layer similarity theory, the friction velocity $u^{*}$ and the sensible heat flux $\left\langle w^{\prime} T^{\prime}\right\rangle$ can be calculated from the mean longitudinal velocity $\langle U\rangle$, surface temperature $T_{s}$, and mean air temperature $\left\langle T_{a}\right\rangle$ using

$$
\begin{gathered}
\langle U\rangle=\frac{u^{*}}{\kappa}\left[\ln \left(\frac{z}{z_{o}}\right)-\Psi_{m}\left(\frac{z}{L}\right)\right] \\
T_{s}-\left\langle T_{a}\right\rangle=\frac{\left\langle w^{\prime} T^{\prime}\right\rangle}{\kappa u^{*}}\left[\ln \left(\frac{z}{z_{o h}}\right)-\Psi_{h}\left(\frac{z}{L}\right)\right]
\end{gathered}
$$

where $z_{o}$ and $z_{o h}$ are roughness lengths for momentum and heat, respectively, and $\Psi_{m}$ and $\Psi_{h}$ are the stability correction functions for momentum and heat, respectively. For bluff rough surfaces, $z_{o h}$ is given by [Brutsaert, 1982]

$$
z_{\text {oh }}=7.4 z_{o} \exp \left(-2.46 z_{o}^{+1 / 4}\right)
$$

where $z_{o}^{+}\left(=u^{*} z_{o} / \nu\right)$ is the roughness Reynolds number and $\nu$ is the kinematic viscosity of air.

Furthermore, for neutral conditions the standard deviations of horizontal velocity $\left(\sigma_{u}\right)$, vertical velocity $\left(\sigma_{w}\right)$, and temperature $\left(\sigma_{T}\right)$ can be calculated from the relations suggested by Kader and Yaglom [1990]: $\sigma_{u}=2.7 u^{*}, \sigma_{w}=1.25 u^{*}$, and $\sigma_{T}=2.9 T^{*}$, where $T^{*}=\left|\left\langle w^{\prime} T^{\prime}\right\rangle\right| / u^{*}$. For unstable conditions, $\sigma_{w}=1.25 u^{*}(1-3 z / L)^{1 / 3}, \sigma_{T}=0.95 T^{*}(-z / L)^{-1 / 3}$ [Wyngaard, 1971]. For stable conditions the near-neutral limits are used [see Sorbjan, 1989, p. 81]. It should be noted that for very stable conditions $(z / L>0.1), \sigma_{u}$ can be larger than expected because of gravity wave occurrence while $u^{*}$ is small. These conditions result in $\sigma_{u} / u^{*}$ that is consistently larger than predictions by Monin and Obukhov [1954] similarity theory. In Figure 6 the measured $\sigma_{u} / u^{*}$ and $\sigma_{w} / u^{*}$ are shown as a function of $z / L$ for the stable stratification case. Notice in Figure 6 that for $z / L>0.1$, three measurements of $\sigma_{u} / u_{*}$ are much larger than 2.7. These three measurements are from the same evening experiment. The similarity theory relationship between $\sigma_{T}$ and $T^{*}$ for stable stability conditions is contaminated by large scatter and is unreliable. This, in part, is due to the near-zero value of the measured sensible heat flux and $T^{*}$. Similar scatter for this relationship is noted in the data presented by Sorbjan [1989, p. 81]. The correlation coefficients $r_{u w}$ and $r_{w T}$ can be calculated from

$$
\begin{gathered}
r_{u w}=\frac{\langle u w\rangle}{\sigma_{u} \sigma_{w}} \\
r_{w T}=\frac{\left\langle w^{\prime} T^{\prime}\right\rangle}{\sigma_{w} \sigma_{T}}
\end{gathered}
$$

Therefore, using (10)-(14), all the necessary parameters (e.g., $u^{*},\left\langle w^{\prime} T^{\prime}\right\rangle, \sigma_{u}, \sigma_{w}, \sigma_{T}, r_{u w}$, and $\left.r_{w T}\right)$ for the pdf's can be calculated from similarity theory. Table 3 shows the compari- 
son of measured and predicted parameters. The $\sigma_{T}$ and $r_{w T}$ for the stable condition and $\sigma_{u}$ and $r_{u w}$ for the unstable condition did not exhibit strong dependence on $z / L$ and thus were not estimated from similarity theory for this experiment. For all other variables, good agreement between measured and predicted moments is noted.

The objective of this study is not to investigate the accuracy of surface layer similarity theory to predict such moments but to demonstrate the usefulness of first- and second-order moments to predict the flux pdf. In our case this was established by using mean meteorological parameters such as $\langle U\rangle, T_{s}$, and $\left\langle T_{a}\right\rangle$ to estimate the flux pdf.
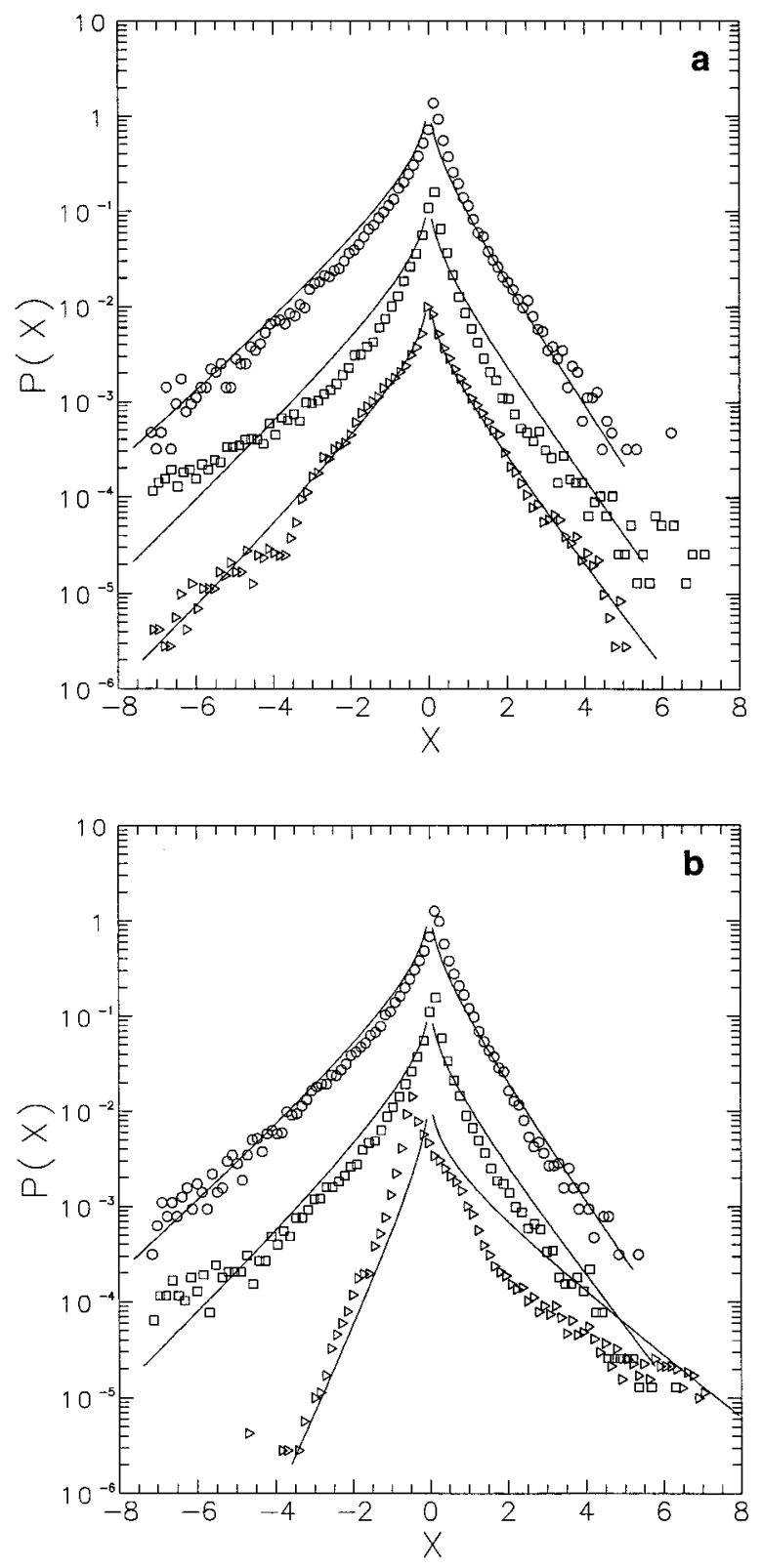

Figure 5. Probability density functions of fluxes under different stability conditions: (a) momentum flux and (b) sensible heat flux. The solid lines are the predictions from (9). Conditions and symbols are the same as Figure 2.

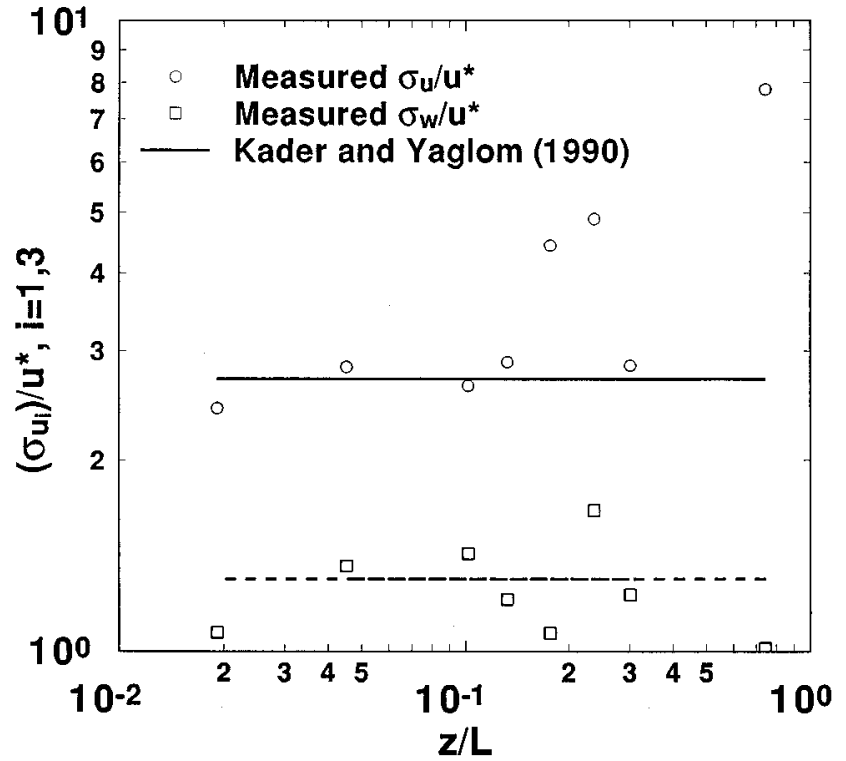

Figure 6. Variation of the normalized standard deviations of the horizontal $\left(\sigma_{u} / u^{*}\right)$ and vertical $\left(\sigma_{w} / u^{*}\right)$ velocity fluctuations for stable stability conditions available during the experiment. Surface layer similarity predictions (solid line is $\sigma_{u} / u^{*}=$ 2.7; dashed line is $\sigma_{w} / u^{*}=1.3$ ) for a neutral ASL from Kader and Yaglom [1990] are also shown.

\section{Conclusion}

The probability density functions were measured for the velocities, temperature, their derivatives, and momentum and sensible heat fluxes in the ASL for a wide range of stability conditions. Our study demonstrated the following:

1. The pdf's for velocity fluctuations are near-Gaussian for a wide range of atmospheric stability conditions. This is consistent with many homogeneous shear flow experiments. However, for stable conditions a slight departure from Gaussian behavior was noted in the vertical velocity. The pdf for temperature fluctuations for neutral and stable conditions followed the velocity pdf distribution. However, for unstable conditions the temperature pdf was found to be non-Gaussian and strongly skewed. This was attributed to the ramp-like pattern in the temperature time series that was absent for neutral and stable stability conditions.

2. The velocity gradient pdf's exhibit long exponential tails with kurtosis close to 6 and are largely independent of atmospheric stratification. For unstable conditions the structure of the temperature gradient pdf confirmed the role of the ramplike structure noted above.

3. For the unstably stratified atmospheric surface layer, organized ramp-like patterns due to forced convection result in a heat transport mechanism that is dissimilar to that of momentum transport. Our measurements showed that for unstable conditions, the kurtosis of temperature was comparable to the velocity gradients. This suggests that the temperature field is being strained by the velocity, as can be expected from the convective transport term in the heat transport equation.

4. For neutral and stable conditions the pdf's of momentum and sensible heat fluxes were well described by the jointGaussian statistics of $u$ and $w$, and $w$ and $T$, respectively. This allowed the use of surface layer similarity theory to predict the necessary statistics for the pdf's of velocities, temperature, and 
fluxes from the mean meteorological parameters. Although the objective of this study is not to investigate the accuracy of surface layer similarity theory, it does demonstrate the usefulness of first- and second-order moments in estimating the flux pdf. The ability to predict the flux pdf from lower-order moments is of great interest in many hydrological and climatic models where the parameterization of flux variability is critical [Hechtel et al., 1990; Mahrt and Ek, 1993].

Acknowledgments. The authors would like to thank Scott Tyler, Teresa Ortenburger, and Mike Mata for their help in the data collection, and National Science Foundation grant EAR-93-04331, California Water Resource Research grant W-812, the Kearney Foundation, and U. C. Davis superfund grant 5 P42ES04699-07 for funding support.

\section{References}

Adrian, R. J., R. T. D. S. Ferreira, and T. Boberg, Turbulent thermal convection in wide horizontal fluid layers, Exp. Fluids, 4, 121-141, 1986.

Albertson, J. D., M. B. Parlange, G. G. Katul, C. R. Chu, and H. Stricker, Sensible heat flux from arid regions: A simple fluxvariance method, Water Resour. Res., 31, 969-973, 1995.

Antonia, R. A., E. J. Hopfinger, Y. Gagne, and F. Anselmet, Temperature structure function in turbulent shear flows, Phys. Rev. A, 30, 2704-2707, 1984.

Balachandar, S., and L. Sirovich, Probability distribution functions in turbulent convection, Phys. Fluids A, 3, 919-927, 1991.

Batchelor, G. K., The Theory of Homogeneous Turbulence, 197 pp., Cambridge Univ. Press, New York, 1953.

Brutsaert, W., Evaporation Into the Atmosphere: Theory, History, and Applications, 300 pp., Kluwer Acad., Norwell, Mass., 1982.

Caughey, S. J., M. Kitchen, and J. R. Leighton, Turbulence structure in convective boundary layers and implications for diffusion, Boundary Layer Meteorol., 25, 345-352, 1983.

Hechtel, L. M., C. H. Moeng, and R. B. Stull, The effects of nonhomogeneous surface fluxes on the convective boundary layer: A case study by large-eddy simulation, J. Atmos. Sci., 47, 1721-1741, 1990.

Jayesh, and Z. Warhaft, Probability distribution, conditional dissipation and transport of passive temperature fluctuations in gridgenerated turbulence, Phys. Fluids A, 4, 2292-2307, 1992.

Kader, B. A., and A. M. Yaglom, Mean fields and fluctuation moments in unstably stratified turbulent boundary layers, J. Fluid Mech., 212, 637-662, 1990.

Kaimal, J. C., and J. J. Finnigan, Atmospheric Boundary Layer Flows: Their Structure and Measurements, 289 pp., Oxford Univ. Press, New York, 1994

Katul, G. G., A model for sensible heat flux probability density function for near-neutral and slightly-stable atmospheric flows, Boundary Layer Meteorol., 71, 1-20, 1994.

Katul, G. G., J. D. Albertson, M. B. Parlange, C. R. Chu, and
H. Stricker, Conditional sampling, bursting, and the intermittent structure of sensible heat flux, J. Geophys. Res., 99, 22,869-22,876, 1994.

Katul, G. G., C. R. Chu, J. D. Albertson, M. B. Parlange, and T. A. Ortenburger, Low-wavenumber spectral characteristics of velocity and temperature in the atmospheric surface layer, J. Geophys. Res., $100,14,243-14,255,1995$ a.

Katul, G. G., M. B. Parlange, J. D. Albertson, and C. R. Chu, Local isotropy and anisotropy in the sheared and heated atmospheric surface layer, Boundary Layer Meteorol., 72, 123-148, 1995b.

Kerr, R. M., Higher-order derivative correlations and the alignment of small scale structure in isotropic numerical turbulence, J. Fluid Mech., 153, 31-53, 1985.

Lesieur, M., Turbulence in Fluids, 412 pp., Kluwer Acad., Norwell, Mass., 1990.

Mahrt, L., and M. Ek, Spatial variability of turbulent fluxes and roughness lengths in HAPEX-MOBILIHY, Boundary Layer Meteorol., 65 , 381-400, 1993.

Metais, M., and M. Lesieur, Spectral large-eddy simulation of isotropic and stably stratified turbulence, J. Fluid Mech., 210, 157-194, 1992.

Monin, A. S., and A. M. Obukhov, Basic laws of mixing in the ground layer of the atmosphere, Trans. Geofiz. Inst. Akad. Nauk. SSSR, 151, 163-187, 1954.

Monin, A. S., and A. M. Yaglom, Statistical Fluid Mechanics, vol. 1, 769 pp., MIT Press, Cambridge, Mass., 1971.

Papoulis, A., Probability, Random Variables and Stochastic Processes, 389 pp., McGraw-Hill, New York, 1972.

Pope, S. B., PDF methods for turbulent reactive flows, Progr. Energy Combust. Sci., 11, 119-192, 1985.

Pumir, A., B. Shraiman, and E. D. Siggia, Exponential tails and random advection, Phys. Rev. Lett., 66, 2984-2987, 1991.

Raupach, M. R., R. A. Antonia, and S. Rajagopalan, Rough wall turbulent boundary layers, Appl. Mech. Rev., 44, 1-25, 1991.

Sorbjan, Z., Structure of the Atmospheric Boundary Layer, 317 pp., Prentice Hall, Englewood Cliffs, N. J., 1989.

Taylor, G. I., The spectrum of turbulence, Proc. R. Soc. London A, 164, 476-490, 1938.

Thoroddsen, S. T., and C. W. Van Atta, Exponential tails and skewness of density-gradient PDF's in stably stratified turbulence, J. Fluid Mech., 244, 547-566, 1992.

Townsend, A. A., The measurement of double and triple correlation derivatives in isotropic turbulence, Proc. Cambridge Philos. Soc., 43, 560 pp., 1947.

Wyngaard, J. C., Local free convection, similarity and the budgets of shear stress and heat flux, J. Atmos. Sci., 28, 1171-1182, 1971.

Wyngaard, J. C., and S. F. Clifford, Taylor's hypothesis and highfrequency turbulence spectra, J. Atmos. Sci., 34, 922-929, 1977.

J. D. Albertson, C. R. Chu, and M. B. Parlange, Hydrologic Science, University of California, Davis, Davis, CA 95616.

G. G. Katul, School of the Environment, Duke University, Box 90328, Durham, NC 27708-0328.

(Received October 18, 1995; revised January 18, 1996; accepted January 23, 1996.) 